\title{
Optical coherence tomography angiography in diabetic retinopathy: a review of current applications
}

\author{
Kai Yuan Tey ${ }^{1}$, Kelvin Teo², Anna C. S. Tan², Kavya Devarajan³, Bingyao Tan², Jacqueline Tan², \\ Leopold Schmetterer ${ }^{3}$ and Marcus Ang ${ }^{4^{*}}$ (D)
}

\begin{abstract}
Background: Diabetic retinopathy (DR) is a leading cause of vision loss in adults. Currently, the standard imaging technique to monitor and prognosticate DR and diabetic maculopathy is dye-based angiography. With the introduction of optical coherence tomography angiography (OCTA), it may serve as a potential rapid, non-invasive imaging modality as an adjunct.

Main text: Recent studies on the role of OCTA in DR include the use of vascular parameters e.g., vessel density, intercapillary spacing, vessel diameter index, length of vessels based on skeletonised OCTA, the total length of vessels, vascular architecture and area of the foveal avascular zone. These quantitative measures may be able to detect changes with the severity and progress of DR for clinical research. OCTA may also serve as a non-invasive imaging method to detect diabetic macula ischemia, which may help predict visual prognosis. However, there are many limitations of OCTA in DR, such as difficulty in segmentation between superficial and deep capillary plexus; and its use in diabetic macula edema where the presence of cystic spaces may affect image results. Future applications of OCTA in the anterior segment include detection of anterior segment ischemia and iris neovascularisation associated with proliferative DR and risk of neovascular glaucoma.
\end{abstract}

Conclusion: OCTA may potentially serve as a useful non-invasive imaging tool in the diagnosis and monitoring of diabetic retinopathy and maculopathy in the future. Future studies may demonstrate how quantitative OCTA measures may have a role in detecting early retinal changes in patients with diabetes.

Keywords: Optical coherence tomography angiography, Fluorescein angiography, Diabetic retinopathy, Screening, Monitoring

\section{Background}

Diabetes is currently on the rise with 422 million of people in the world reported to have diabetes in 2014 [1] and is a systemic disease with a multitude of complications which may involve the eyes. The most common ocular complication is diabetic retinopathy (DR), which may be asymptomatic in the early stages, however, disease progression can lead to severe vision loss [2]. Diabetic retinopathy is a leading cause of blindness in working age

\footnotetext{
* Correspondence: Marcus.Ang@Singhealth.com.sg

${ }^{4}$ Singapore National Eye Centre, 11 Third Hospital Ave, Singapore 168751; Duke-NUS Medical School, 8 College Rd, Singapore 169857, Singapore Full list of author information is available at the end of the article
}

adults [3] and is estimated to affect 1 in 3 diabetic patients $[4,5]$. Diagnosis of DR is based on clinical findings and can be divided into 2 categories - early non-proliferative diabetic retinopathy (NPDR) and more advanced proliferative diabetic retinopathy (PDR) associated with retinal ischemia and development of neovascularisation [6]. The main sight-threatening complications of DR are diabetic maculopathy, which include diabetic macular oedema (DME) and diabetic macular ischemia (DMI) [7], and complications from PDR - vitreous haemorrhage and retinal detachment [8]. Digital retinal fundus image analysis has been shown to be able to detect early DR and DME in routine DR screening [9-11]. While it has high

(c) The Author(s). 2019 Open Access This article is distributed under the terms of the Creative Commons Attribution 4.0 International License (http://creativecommons.org/licenses/by/4.0/), which permits unrestricted use, distribution, and 
sensitivity and specificity, it has been shown to have a low negative predictive value [11].

Optical Coherence Tomography (OCT) offers a noninvasive, rapid imaging modality that can provide imaging of the cross-sectional structures of the retina by using low-coherence interferometry to capture high resolution two dimensional images from the optical scattering from different layers of the retina [12] and is an essential tool in the detection and monitoring of DME [13], and DMI with inner retinal thinning [14]. Optical coherence tomography angiography (OCTA) is a novel use of OCT to visualise the microvasculature of the retina and choroid without the need for dye injection [15]. This is performed through repeated scans at the same location to detect the changes in OCT reflectance signal from the flow through blood vessels [16, 17]. It allows depth-resolved imaging of the retinal vasculature and is an ideal approach for various retinal conditions such as DR, retinal venous occlusion, uveitis, retinal arterial occlusion and age-related macular degeneration $[18,19]$.

In this review, we will discuss the role of OCTA in the evaluation and monitoring of DR, diabetic maculopathy and the anterior segment involvement in DR.

\section{Main text}

\section{Literature search}

We conducted a literature search via PUBMED database for articles written in the English language until January 1, 2019, with the following medical subject headings: "OCTA," "OCT angiography," "Diabetic Retinopathy," or "Diabetes". All papers that used OCTA were reviewed for findings in DR and bibliographies were hand-searched for further studies. Eighty-eight articles were identified, with 11 papers being excluded as they were either reviews, inter-instrumental reliability study or case report/series. There was a total of 58 prospective studies, of which 17 were observational, 30 were observational and cross-sectional, and 11 were observational case-control study. There was a total of 19 retrospective studies, of which 12 were observational, two were observational cross-sectional, two were case-control and three were cross-sectional. In total, there were two multi-centred studies. The number of patients vary widely among studies. In addition to that, we also performed an additional search via PUBMED database with the following medical subject headings: "OCTA", "Anterior Segment", which returned 27 articles, of which three articles were excluded as they were either reviews or case-report.

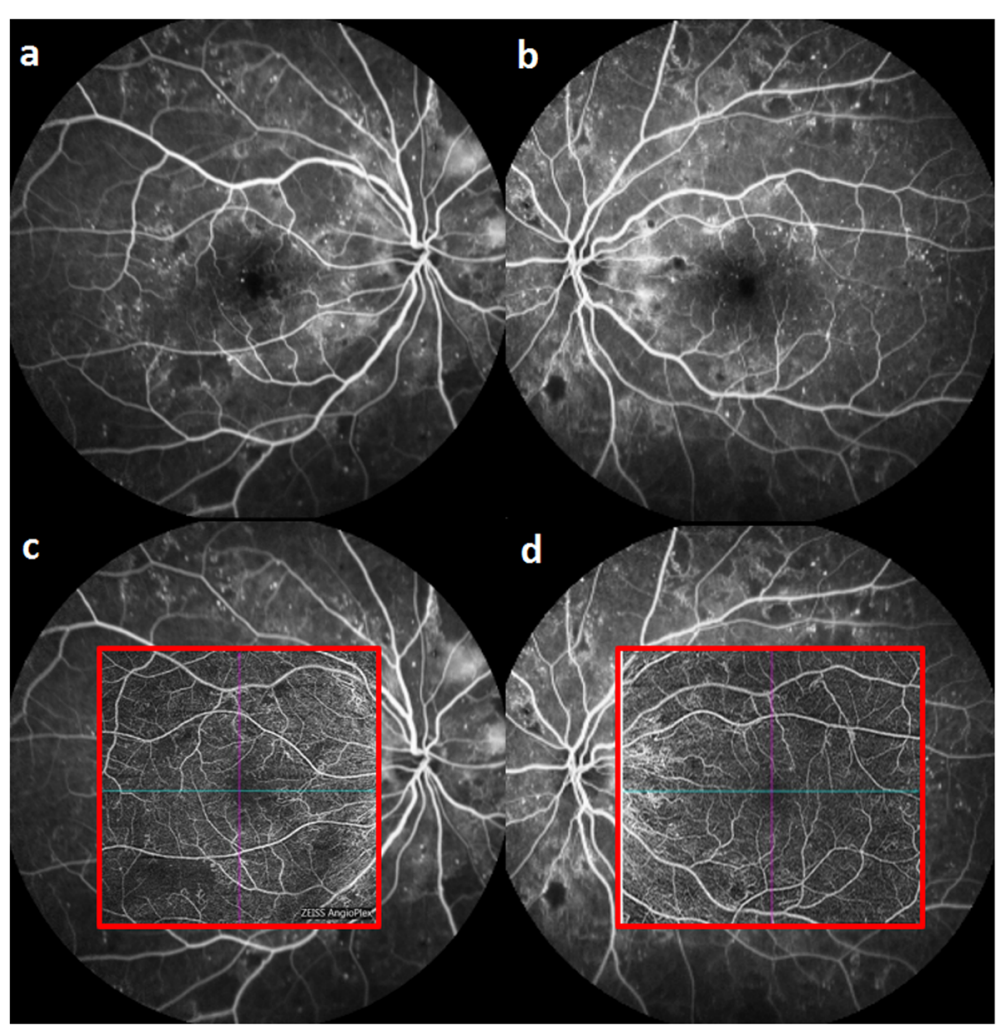

Fig. 1 Comparison of Fluorescein Angiography and OCTA. a \& b Fluorescein angiography images of a patient with proliferative diabetic retinopathy. These FA images show patchy areas of capillary drop out and presence of neovascularizations elsewhere (NVE). c \& d Corresponding OCTA images (generated via ZEISS AngioFlex) of (a) and (b) being superimposed on the FA images. The OCTA images also show areas of capillary drop out and new vessels without leakage 


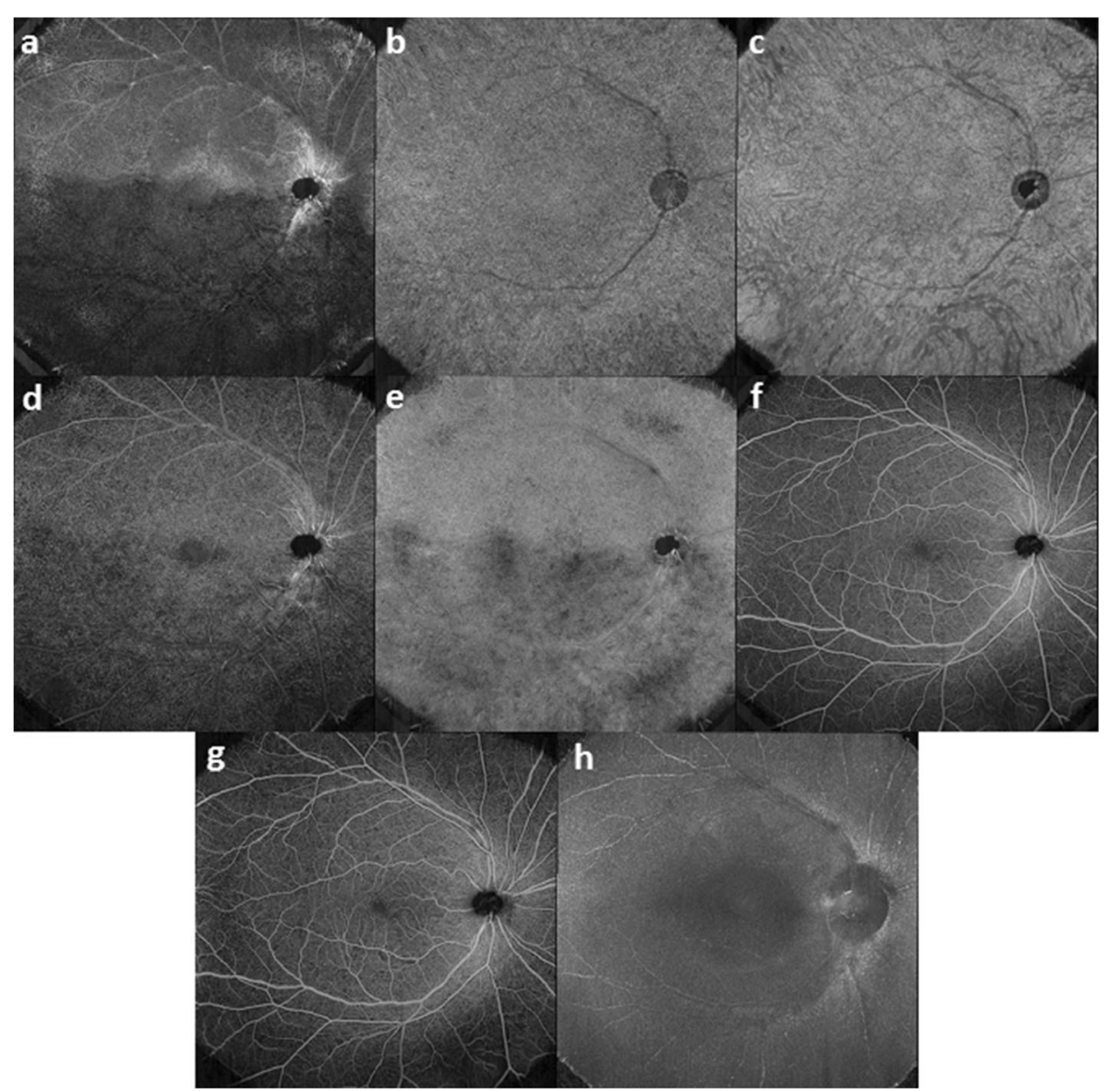

Fig. 2 A series of montaged OCTA in patients with diabetic retinopathy (DR). This is a series of montaged OCTA images $15 \mathrm{~mm} \times 15 \mathrm{~mm}$ taken at different segment in the right eye of a male (a-h) with DR. a Foveal avascular zone; b Choriocapillaris; c Choroid; $\mathbf{d}$ Deep capillary plexuses; e Outer-retina-choroid complex; $\mathbf{f}$ Retina; $\mathbf{g}$ Superficial capillary plexuses; $\mathbf{h}$ Vitreoretinal interface

\section{Fluoresceine angiography and optical coherence tomography}

Fluorescein angiography (FA) is helpful in the evaluation of the retinal vasculature and was first described in 1961 and later adopted as a standard practice in the field of Ophthalmology [20]. Fluorescein angiography can be used to evaluate the retinal vasculature to monitor the progression of DR and DME [21, 22]. In FA, sodium fluorescein is injected intravenously and with the use of excitation and barrier filters, high contrast en face images of the retinal vasculature can be visualised [23]. The advantage of FA lies in its ability to assess properties such as perfusion (e.g., arm-retinal time, arterio-venous transit), leakage and staining [24]. Flash photography and recently, scanning laser ophthalmoscopy can be used to capture FA images to allow visualisation of the retinal vessels in high contrast $[25,26]$. With ultra-widefield FA, the imaging field can visualise the entire posterior segment and extend beyond the equator of the eye, giving a field of view of up to 200 degrees [27].
FA is a primary en face modality, and cross-sectional segmentation of the retinal vessels is not possible [28]. Depth resolution is inferred from FA, and indocyanine green angiography (ICGA) can be used to differentiate choroidal from retinal perfusion as it has a larger molecule size [28].

On the other hand, OCTA has several advantages over dye angiography in terms of acquisition speed and imaging information (Fig. 1) [28]. OCTA images are essentially motion-contrast images with images obtained via multiple $\mathrm{B}$ scans at the same location, and information derived is based on the backscattering of light from the changes in the intensity and phase from each scan changes due to blood flow while the neurosensory tissue will remain stationary, henceforth this approach eliminates the need for dye. The primary advantage of OCTA is the ability to obtain depth-resolved imaging of the retinal vasculature [29]. It is able to generate the images of the superficial and deep retinal layers by default [30] and this can be modified to further segment the retinal vasculature and provide images of other layers such 


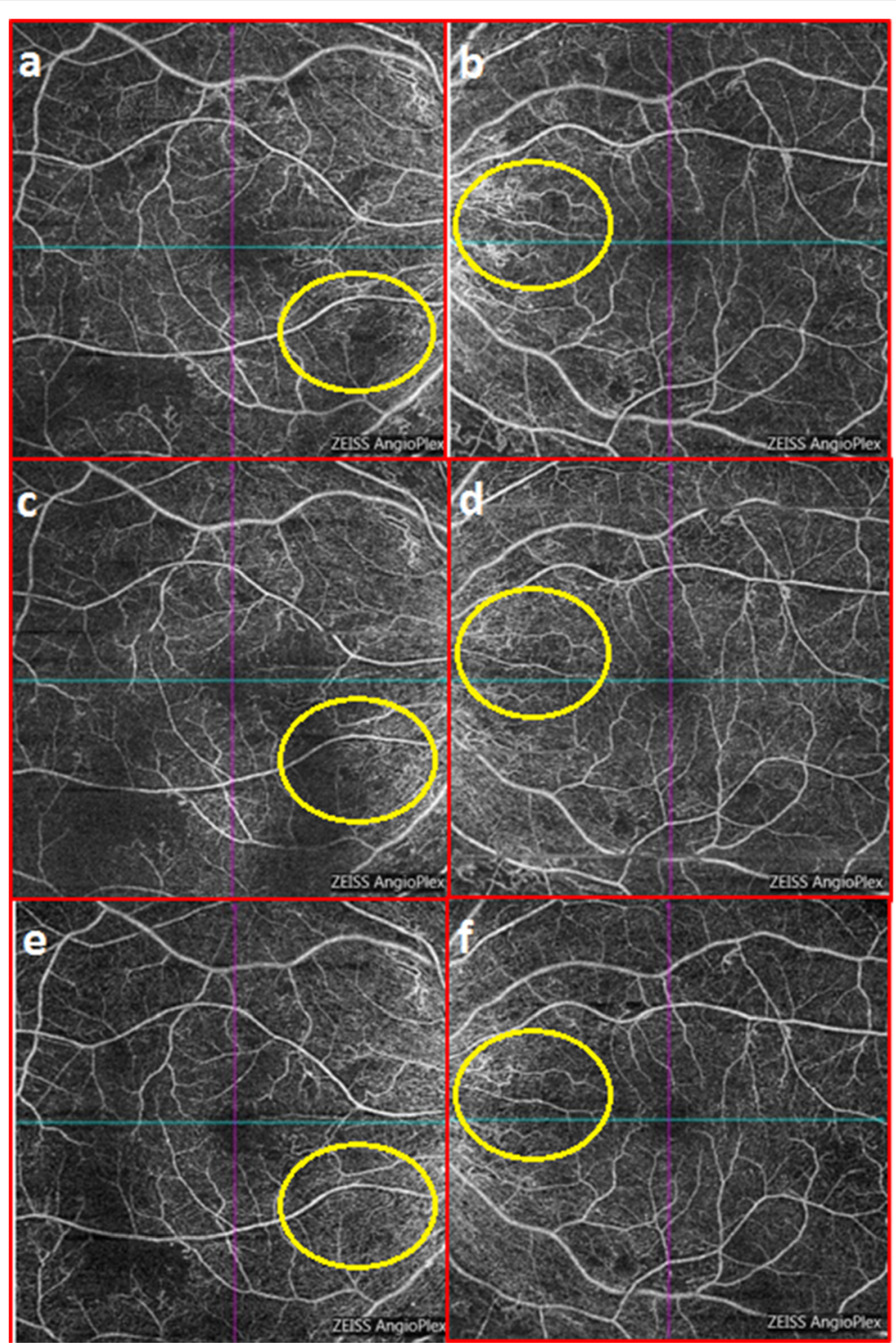

Fig. 3 Monitoring of treatment outcome in patients with proliferative diabetic retinopathy using OCTA. This is a series of OCTA images of a 26 years old female with proliferative diabetic retinopathy taken at baseline $(\mathbf{a} \& \mathbf{b}), 1$ st month $(\mathbf{c} \& \mathbf{d})$ and 6 th month $(\mathbf{e} \& \mathbf{f})$ post IVT treatment (bevacizumab). OCTA is able to detect changes - NVE regression is noted

as the radial peripapillary network and choriocapillaris [28, $31,32]$, which can help to visualise pathological features that are not previously seen in 2-layers segmentation [32]. The corresponding flow signal on OCT B-scans allows crosssectional localization of the vasculature in question.

There are several shortcomings for OCTA use. Firstly, the field of view of OCTA is narrower than FA, with most images being $3 \mathrm{~mm}$ by $3 \mathrm{~mm}$ [28]. The largest scanning area that is achievable with commercially available OCTA devices is $8 \mathrm{~mm}$ by $8 \mathrm{~mm}$ which grants a field of view of approximately 30 degrees [33]. Thus, OCTA has poor ability in generating good quality peripheral retinal images [28]. Even with the introduction of wide-field OCTA that is able to generate images of $12 \mathrm{~mm}$ by $12 \mathrm{~mm}$ the field of view is still not comparable to standard and ultra wide-field FA/ICGA [34, 35]. To overcome this limitation, the montaging algorithm has been introduced which allows the $12 \mathrm{~mm}$ by $12 \mathrm{~mm}$ images to be montaged and generate a wider field of view [28]. This approach, however, results in an increase of scan acquisition time, and inherent inaccuracies due to misalignment of images [36]. Secondly, OCTA is unable to assess dynamic characteristics of flow velocity, and leakage which is sometimes necessary to assess various retina pathology. Thirdly, processing of high-resolution images can be time-consuming [37] and images 
generated via OCTA are highly susceptible to projection artefacts due to the presence of the superficial blood flows resulting in difficulty in interpreting the deep retinal vasculatures [38]. While this can be corrected via projection removal algorithms, this method may potentially result in loss of flow information within the deeper layer, giving a disjointed image [39]. Additionally, OCTA images are prone to motion artefacts as well, which often appear as a white line across the image, and can be improved with motion correction function and eye-tracking algorithm [40].

\section{Morphological changes of DR on OCTA}

Several morphological changes of DR can be detected by OCTA - microaneurysms (MAs), intraretinal microvascular abnormalities (IRMAs), nonperfusion areas and neovascularizations (NVs) [41], and it is able to offer additional information with respect to the localization of these changes [42].

Microaneurysms are lesions that often manifest in early DR. Thompson et al. showed that OCTA is able to pick up MAs, not otherwise shown on a dilated clinical examination [43]. OCTA is able to localize MAs precisely [42]. There are, however, discrepancies, among studies, in regards to the detectability of MAs between FA and OCTA $[42,44-46]$. FA has demonstrated higher sensitivity compared to OCTA [47-49]. On the other hand, the majority of MAs detected by OCTA has a corresponding finding in FA [45]. Schwartz et al. and Ishibazawa et al. demonstrated that OCTA can detect MAs that are otherwise not detectable on FA $[42,46]$. Detection of MAs using OCTA, however, may be influenced by blood flow turbulence within the MAs [50] and hence the discrepancy found among the studies [41, 48, 51, 52]. Parravano et al. have identified a correlation between the MAs' reflectivity and its detectability on OCTA - MAs that are hyper-reflective are more likely to be detected but this may also be affected by turbulent blood flow in MAs [53]. As such, it is still unclear if OCTA is comparable to FA in terms of detecting MAs.

Intraretinal microvascular abnormalities are shunt vessels due to abnormal branching or dilation of existing capillaries within the retina that help to supply areas of non-perfusion in DR. Visualisation of IRMAs has been made possible with OCTA via the use of en face images and are shown as dilated or looping vessels near the areas of capillary loss, and has a higher detection rate on OCTA than color fundus photography [54]. The use of OCTA also allows identification of other features such as the presence of intraretinal hyperreflective dots and outpouching of the internal limiting membrane (ILM) [55], which may be useful in detection of IRMAs.

Retinal NVs are detectable on OCTA via observation of flow signal above the ILM [55]. OCTA can detect early retinal NVs [49] and identify the origins and morphological patterns of NVs in PDR, hence allowing classification of the lesion, offering a better understanding of the pathophysiology and helps to guide the management strategies [56]. OCTA is also able to detect subtle $\mathrm{NVs}$, which is difficult to differentiate from a MAs on FA [49].

Owing to OCTA's ability to segment the various layer of the retina, it is able to distinguish retinal NVs from IRMAs, which may not always be possible on FA or clinical examination [44], and is of importance as very often, retinal NVs may form next to IRMA [55]. In addition to that, de Carlo et al. showed that retinal NVs often appear next to retinal non-perfusion areas [55]. As such, OCTA may be useful in helping us to differentiate NPDR from $\mathrm{DR}$, and aid us in following up and management planning.

\section{Quantitative measures in OCTA and its application in DR}

Various quantitative measures have been developed over the years to aid research studies as well as the understanding of DR pathophysiology. These quantitative measurements have been shown to allow objective identification and staging of NPDR - mild, moderate and severe, with significant diagnostic accuracy and predictability of DR progression [57]. To the best of our knowledge, we are not aware of any normal data material available for the different OCTA measurements. Several OCTA vascular quantitative measures currently used in research and has yet to be adopted in clinical practice have been proposed:

1) The area filled by binarized vessels (vessel area density - VD or vessel perfusion density - PD) [57-60];

2) Vessel spacing/inter-capillary area [61];

3) Length of the blood vessel based on the skeletonized OCTA (vascular length density - VLD or skeleton density - SD) [57, 59];

4) Vessel diameter index (VDI) [57];

5) Total length of vessels (vessel length fraction) [62];

6) Vascular architecture and branching, (vessel tortuosity and fractal dimension - FD) [58];

7) Area of the foveal avascular zone - FAZ [63].

Certain commercially available devices - Topcon DRIOCT Triton Swept-source OCT, Optovue RTVue-XR, Heidelberg-Engineering and Zeiss Cirrus 5000-HD-OCT enhance efficiency and reduce bias as they automatically map VD, FAZ and PD [64, 65]. In general, VD, SD, FD and VDI are highly reproducible among graders and studies have found that vascular changes in DR may be characterized by these parameters [66].

\section{Vessel density}

Vessel density is defined as the proportion of blood vessel area over the total measured area [67]. Measurements of VD are highly reproducible and comparisons 
of measurement should be made using the same device [67]. This parameter varies with age and sex, and should be taken into consideration when interpreting the results [68]. Vessel density also changes with retinal structural characteristics including retinal thickness and volume, and a reduced VD would correlate with thinner macular ganglion cell or inner plexiform layer [69]. Vessel density decreases in both the DCP and SCP of a patient with DR [70], as well as a diabetic patient without DR, attributing to the fact that parafoveal capillary nonperfusion in DCP may potentially be an early sign of DR [70-73].

Vessel density in DCP may predict DR severity and identify patients at risk as it is able to detect retinal vascular changes in diabetic patients without any signs of DR [71, 74]. Vascular spacing and alterations in VD in SCP, however, have found to have a stronger correlation with the severity of $\mathrm{DR}$ as compared to VD in DCP, PD in SCP or FAZ area [68, 70, 71]. Despite the contradictory results, VD has shown to decrease in both DCP and SCP in DR, and hence able to assist in predicting treatment outcome along with following up of patients (Fig. 2) [71, 74].

\section{Inter-capillary spacing}

Inter-capillary spacing can be detected by areas that are not perfused and occur much earlier than VD changes [60]. Bhanushali et al. found that large vessel spacing, especially those in the SCP, are more sensitive than VD and FAZ area in the diagnosis of DR and it reflects the severity of DR [74]. The extrafoveal avascular area may help to distinguish early NPDR from healthy eyes [75]. Schottenhamml et al. found that inter-capillary spacebased algorithm is more sensitive than vascular densitybased methods to calculate early capillary drop-out or non-perfusion areas [61]. As capillary non-perfusion area enlarges with progression in severity of DR, the quantitative analysis of retinal non-perfusion on OCTA may be useful for early detection and monitoring of disease in patients with diabetes and DR [76].

\section{Vascular architecture and branching - vessel tortuosity and fractal dimension}

Vessel tortuosity is a quantitative measure from fundus images via computer-assisted software and is defined as the integral of the curvature square along the path of vessel, normalized by the total path length [77]. Patients with diabetes have been found to have increased vessel tortuosity as compared to healthy controls and are related to mild and moderate stages of DR, suggesting that vessel tortuosity may be an early indicator of microvascular damage in the retina [78]. Vessel tortuosity may be used to distinguish moderate to severe NPDR from PDR, particularly in the SCP region. FAZ area and acircularity correlate with vessel tortuosity in $3 \mathrm{~mm}^{2}$ and 1.5 $\mathrm{mm}^{2}$ of SCP. As this parameter increases with worsening of NPDR and decreases in PDR, it may serve as a quantitative marker to monitor the progression of DR [58].

Fractal dimension is a measure of the complexity of a vasculature branching pattern [79] and is derived from applying fractal analysis to OCTA images [80]. Fractal dimension was found to be an early indicator of DR [81] and was reduced in both SCP and DCP in patients with diabetes compared to healthy controls, with a greater reduction in the DCP $[82,83]$.

\section{Foveal avascular zone assessment}

Johannesen et al. [84] conducted a systemic review on 8 studies investigating the changes in the FAZ in DR patients. Seven of these studies found that the FAZ in NPDR patients will be larger as compared with the healthy control group. Six studies on OCTA in DR found that patients with PDR have a larger FAZ as compared to the control group, and a decrease in foveal capillary perfusion in diabetics compared to controls. This increase in FAZ with the progression of DR may indicate increasing non-perfusion [85].

\section{Use of OCTA in macula disease in DR}

Diabetic macular ischemia is characterized by the occlusion and loss of the macular capillary network or capillary dropout [86]. A study showed that non-perfused areas in DCP and reduced VD reflect the macular photoreceptor disruption in DMI $[86,87]$. In the area of the disrupted ellipsoid zone of the photoreceptor, choroidal circulation (CC) layer had greater areas of flow void and hence alteration of $\mathrm{CC}$ appears to play a role in the pathogenesis of DR and DMI [88]. Wide-field OCTA images have shown that large arterioles situated in both superficial and deep layers seem to be the perfusion boundaries, which may serve as a novel anatomic factor to predict the likelihood of non-perfusion development (Fig. 3) [89] While FA is the gold standard for diagnosing DMI, OCTA may be able to do so as well $[21,86]$ since OCTA may provide images with higher details with respect to macular status [86] and high intergrader agreement [21]. Vascular quantitative measures of OCTA have also shown to be able to help screen and monitor DMI in patients with no clinical evidence of DR [90]. With further advancement in the technology, OCTA may serve as an alternative noninvasive method to FA to detect DMI and help predict visual prognosis.

Diabetic macular edema refers to the accumulation of fluid in the macula due to leaking blood vessels. While OCT can illustrate structural changes prominently and help in the detection of these cystic spaces [91], OCTA has low reliability in visualizing the DCP in patients with DME [92]. The accumulated fluid may interfere with 
imaging and segmentation capabilities of OCT as accurate identification of anatomical landmarks is needed for the complex automated process needed for correct segmentation, and incorrect segmentation may affect OCTA images [36]. DME has an inverse relationship with OCTA signal intensity [93] because the fluid weakens the reflected signal from deeper layers [94]. Spaide et al. reported that the rate of flow voiding does not match with the cystic space exactly as the vessels may be compressed by the cystic space or fluid may pool in the region of low flow rate in the DCP [95].

Regardless, Lee et al. overcame the segmentation issues by carefully adjusting the boundary between the SCP and DCP in the eyes with severe DME, and demonstrated that patients with DME exhibit significant damage to the integrity of the DCP but not the SCP [92]. It was also demonstrated that OCTA was able to assist us in quantifying macular perfusion [96] and measuring the FAZ in patients with DME [96, 97]. Using an inner segmentation of the inner retinal border and an outer segmentation of the retinal pigment epithelium, details of the macula perfusion can still be obtained in the presence of DME even though it may be difficult to differentiate between the SCP and DCP [40].

\section{Anterior segment optical coherence tomography angiography in diabetes mellitus}

Healthy iris vasculature comprises a major arterial circle that is supplied by the anterior and long posterior ciliary arteries, and a minor arterial circle found along the border of the pupil linked by radially oriented vessels within the iris stroma. In severe stages of DR, new vessels are not confined to the retina; these can grow around the pupillary border, the root of iris and can penetrate the anterior surface of the iris in severe cases. This is known as iris neovascularization (NVI) or rubeosis which can lead to the potentially sight-threatening complication of neovascular glaucoma (NVG) [98]. It is crucial to detect NVI in its early stage as prompt treatment may prevent NVG. This complication is usually diagnosed clinically by gonioscopy and although FA may help, this is not frequently the modality of choice. A potential alternative is the use of OCTA adapted for the anterior segment [99]. While current commercially available OCTA is designed to examine the posterior segment of the eye, an adapter lens can be used to provide high-quality images of the anterior segment vasculature with a good inter-observer agreement for qualitative measurements [100]. Early studies demonstrated a method of obtaining OCTA images of the cornea and limbal vasculature with great consistency [101] and allow us to compare normal and diseased iris vessels in the detection of NVI [102].

Adapting OCTA for anterior segment does come with several downsides. Specialised anterior segment adaptive lenses have to be used $[57,101]$ and current software are meant for imaging the posterior segment, therefore resulting in non-parallel segmentation and artefacts due to the curvature of the cornea [103]. Anterior segment OCTA is incapable of registering scans and providing localization required for comparison of serial scans [100, 104]. In addition, motion artefacts are common in anterior scans due to a lack of motion correction software [105].

Furthermore, anterior segment OCTA is not able to visualise deeper vessels in eyes with corneal opacities, dense iris pigmentation, or vessels in thick iris tumours. It has poor detection of vessels with minimal flow since the flow of erythrocytes are slower in small calibre vessels and may be below the detection threshold. Since OCTA are optimized for the posterior segment which has mainly traversing blood flows in the vessels, anterior segment vessels with axial flow may not be detected [106].

\section{Conclusion}

OCTA may potentially serve as a good alternative in the diagnosis and monitoring of diabetic retinopathy and maculopathy due to its non-invasiveness nature. However, the current quantitative measures developed have been more useful in research studies and their clinical implications are not yet well established. At the moment, these measures are not necessary for the diagnosis and monitoring of DR and its associated complications as there are existing methods that are clinically proven to be useful. However, with more studies being done in the near future, these quantitative OCTA measures may have a role in detecting subclinical disease. Anterior segment OCTA, especially in the imaging of the iris, may also be a useful biomarker in monitoring the progression of DR and potentially prevent severe complications.

\section{Abbreviations}

CC: Choroidal circulation; DCP: Deep Capillary Plexuses; DME: Diabetic Macula Edema; DMI: Diabetic Macula Ischemia; DR: Diabetic retinopathy; FA: Fluorescein angiography; FAZ: Foveal avascular zone; FD: Fractal dimension; ICGA: Indocyanine green angiography; ILM: Internal limiting membrane; IRMAs: Intraretinal microvascular abnormalities; IVT: Intravitreal Therapy; MAs: Microaneurysms; NPDR: Non-proliferative diabetic retinopathy; NVE: Neovascularization Elsewhere; NVG: Neovascular glaucoma; NVI: Iris neovascularization; NVs: Neovascularizations; OCT: Optical Coherence Tomography; OCTA: Optical Coherence Tomography Angiography; PD: Vessel perfusion density; PDR: Proliferative diabetic retinopathy; SCP: Superficial Capillary Plexuses; SD: Skeleton density; VD: Vessel area density; VDI: Vessel diameter index; VLD: Vascular length density

\section{Acknowledgements}

Not applicable

Authors' contributions

All authors contributed equally in this work. All authors read and approved the final manuscript.

Funding

Not applicable 


\section{Competing interests}

The authors declare that they have no competing interests.

\section{Author details}

'Hobart Clinical School, Level 3, 43 Collins Street, Hobart, TAS 7000, Australia. ${ }^{2}$ Singapore National Eye Centre, 11 Third Hospital Ave, Singapore 168751, Singapore. ${ }^{3}$ Singapore Eye Research Institute, 20 College Road Discovery Tower, Level 6 The Academia, Singapore 169856, Singapore. ${ }^{4}$ Singapore National Eye Centre, 11 Third Hospital Ave, Singapore 168751; Duke-NUS Medical School, 8 College Rd, Singapore 169857, Singapore.

\section{Received: 23 April 2019 Accepted: 14 October 2019}

Published online: 18 November 2019

\section{References}

1. World Health Organisation. Global report on diabates. Geneva: World Health Organisation; 2016.

2. Fong DS, Aiello L, Gardner TW, King GL, Blankenship G, Cavallerano JD, et al. Diabetic retinopathy. Diabetes Care. 2003:26(suppl 1):s99-s102.

3. Engelgau MM, Geiss LS, Saaddine JB, Boyle JP, Benjamin SM, Gregg EW, et al. The evolving diabetes burden in the United States. Ann Intern Med. 2004;140(11):945-50.

4. Roy MS, Klein R, O'Colmain BJ, Klein BE, Moss SE, Kempen JH. The prevalence of diabetic retinopathy among adults in the United States. Arch Ophthalmol. 2004;122(4):546-51.

5. Zhang X, Saaddine JB, Chou C-F, Cotch MF, Cheng YJ, Geiss LS, et al. Prevalence of diabetic retinopathy in the United States, 2005-2008. JAMA. 2010;304(6):649-56.

6. Kumar V, Abbas AK, Fausto N, Aster JC. Diabetes mellitus. In: Schmitt W, Gruliow R, editors. Robbins and Cotran pathologic basis of disease. 8th ed. Philadelphia: Elsevier Inc; 2007. p. 775-87.

7. Mohamed Q, Gillies MC, Wong TY. Management of diabetic retinopathya systematic review. JAMA. 2007;298(8):902-16.

8. Nentwich MM, Ulbig MW. Diabetic retinopathy - ocular complications of diabetes mellitus. World J Diabetes. 2015;6(3):489-99.

9. Saari JM, Summanen P, Kivelä T, Saari KM. Sensitivity and specificity of digital retinal images in grading diabetic retinopathy. Acta Ophthalmol Scand. 2004;82(2):126-30.

10. Goh JK, Cheung CY, Sim SS, Tan PC, Tan GS, Wong TY. Retinal imaging techniques for diabetic retinopathy screening. J Diabetes Sci Technol. 2016; 10(2):282-94.

11. D'Aloisio R, Giglio R, Di Nicola M, De Giacinto C, Pastore MR, Tognetto D, et al. Diagnostic accuracy of digital retinal fundus image analysis in detecting diabetic maculopathy in type 2 diabetes mellitus. Ophthalmic Res. 2019;61(2):100-6.

12. Youngquist RC, Carr S, Davies DE. Optical coherence-domain reflectometry: a new optical evaluation technique. Opt Lett. 1987;12(3):158-60.

13. Virgili G, Menchini F, Casazza G, Hogg R, Das RR, Wang X, et al. Optical coherence tomography $(\mathrm{OCT})$ for detection of macular oedema in patients with diabetic retinopathy. Cochrane Database Syst Rev. 2015;1:CD008081.

14. Sim DA, Keane PA, Fung S, Karampelas M, Sadda SR, Fruttiger M, et al. Quantitative analysis of diabetic macular ischemia using optical coherence tomographyoct features of diabetic macular ischemia. Invest Ophthalmol Vis Sci. 2014;55(1):417-23.

15. Fingler J, Readhead C, Schwartz DM, Fraser SE. Phase-contrast OCT imaging of transverse flows in the mouse retina and choroid. Invest Ophthalmol Vis Sci. 2008;49(11):5055-9.

16. Hagag AM, Gao SS, Jia Y, Huang D. Optical coherence tomography angiography: Technical principles and clinical applications in ophthalmology. Taiwan J Ophthalmol. 2017;7(3):115-29.

17. Ang M, Tan ACS, Cheung CMG, Keane PA, Dolz-Marco R, Sng CCA, et al. Optical coherence tomography angiography: a review of current and future clinical applications. Graefes Arch Clin Exp Ophthalmol. 2018;256(2):237-45.

18. Agarwal A, Grewal DS, Jaffe GJ, Stewart MW, Srivastava S, Gupta V. Current role of optical coherence tomography angiography: Expert panel discussion. Indian J Ophthalmol. 2018;66(12):1696-9.

19. Tan ACS, Tan GS, Denniston AK, Keane PA, Ang M, Milea D, et al. An overview of the clinical applications of optical coherence tomography angiography. Eye (Lond). 2018;32(2):262-86.

20. Gass JDM, Sever RJ, Sparks D, Goren J. A combined technique of fluorescein funduscopy and angiography of the eye. Arch Ophthalmol. 1967;78(4):455-61.
21. Bradley PD, Sim DA, Keane PA, Cardoso J, Agrawal R, Tufail A, et al. The evaluation of diabetic macular ischemia using optical coherence tomography angiography OCTA in diabetic retinopathy. Invest Ophthalmol Vis Sci. 2016;57(2):626-31.

22. Salz DA, Witkin AJ. Imaging in diabetic retinopathy. Middle East Afr J Ophthalmol. 2015;22(2):145-50.

23. Kim DY, Fingler J, Zawadzki RJ, Park SS, Morse LS, Schwartz DM, et al. Optical imaging of the chorioretinal vasculature in the living human eye. Proc Natl Acad Sci U S A. 2013;110(35):14354-9.

24. Spaide RF, Klancnik JM Jr, Cooney MJ. Retinal vascular layers imaged by fluorescein angiography and optical coherence tomography angiography. JAMA Ophthalmol. 2015;133(1):45-50.

25. Shiraki K, Moriwaki M, Yanagihara N, Kohno T, Miki T. Indocyanine green angiograms of choroidal nevi: comparison between confocal and nonconfocal scanning laser ophthalmoscope and fundus video camera. Jpn J Ophthalmol. 2001;45(4):368-74.

26. Bartsch DU, Weinreb RN, Zinser G, Freeman WR. Confocal scanning infrared laser ophthalmoscopy for indocyanine green angiography. Am J Ophthalmol. 1995;120(5):642-51.

27. Klufas MA, Yannuzzi NA, Pang CE, Srinivas S, Sadda SR, Freund KB, et al. Feasibility and clinical utility of ultra-widefield indocyanine green angiography. Retina. 2015;35(3):508-20.

28. de Carlo TE, Romano A, Waheed NK, Duker JS. A review of optical coherence tomography angiography (OCTA). Int J Retina Vitreous. 2015;1:5.

29. Chua J, Chin CWL, Tan B, Wong SH, Devarajan K, Le TT, et al. Impact of systemic vascular risk factors on the choriocapillaris using optical coherence tomography angiography in patients with systemic hypertension. Sci Rep. 2019;9(1):5819.

30. Garrity ST, lafe NA, Phasukkijwatana N, Chen X, Sarraf D. Quantitative analysis of three distinct retinal capillary plexuses in healthy eyes using optical coherence tomography angiographyquantitative analysis using OCTA. Invest Ophthalmol Vis Sci. 2017;58(12):5548-55.

31. Guo Y, Camino A, Zhang M, Wang J, Huang D, Hwang T, et al. Automated segmentation of retinal layer boundaries and capillary plexuses in wide-field optical coherence tomographic angiography. Biomed Opt Express. 2018; 9(9):4429-42.

32. Hwang TS, Zhang $M$, Bhavsar $K$, Zhang $X$, Campbell JP, Lin $P$, et al. Visualization of 3 distinct retinal plexuses by projection-resolved optical coherence tomography angiography in diabetic retinopathy. JAMA Ophthalmol. 2016;134(12):1411-9.

33. Salas M, Augustin M, Felberer F, Wartak A, Laslandes M, Ginner L, et al. Compact akinetic swept source optical coherence tomography angiography at $1060 \mathrm{~nm}$ supporting a wide field of view and adaptive optics imaging modes of the posterior eye. Biomed Opt Express. 2018;9(4):1871-92.

34. Tian M, Tappeiner C, Zinkernagel MS, Huf W, Wolf S, Munk MR. Evaluation of vascular changes in intermediate uveitis and retinal vasculitis using sweptsource wide-field optical coherence tomography angiography. $\mathrm{Br} J$ Ophthalmol. 2019:103(9):1289-95.

35. Sawada O, Ichiyama Y, Obata S, Ito Y, Kakinoki M, Sawada T, et al. Comparison between wide-angle OCT angiography and ultra-wide field fluorescein angiography for detecting non-perfusion areas and retinal neovascularization in eyes with diabetic retinopathy. Graefes Arch Clin Exp Ophthalmol. 2018;256(7):1275-80.

36. Sorour $\mathrm{O}$, Arya M, Waheed $\mathrm{N}$. New findings and challenges in OCT angiography for diabetic retinopathy. Ann Eye Sci. 2018;3(8):44-44.

37. Pichi F, Sarraf D, Morara M, Mazumdar S, Neri P, Gupta V. Pearls and pitfalls of optical coherence tomography angiography in the multimodal evaluation of uveitis. J Ophthalmic Inflamm Infect. 2017;7(1):20.

38. Zhang Q, Lee CS, Chao J, Chen CL, Zhang T, Sharma U, et al. Wide-field optical coherence tomography based microangiography for retinal imaging. Sci Rep. 2016;6:22017.

39. Nesper PL, Soetikno BT, Zhang HF, Fawzi AA. OCT angiography and visiblelight OCT in diabetic retinopathy. Vis Res. 2017;139:191-203.

40. Sambhav K, Grover S, Chalam KV. The application of optical coherence tomography angiography in retinal diseases. Surv Ophthalmol. 2017;62(6): 838-66

41. Matsunaga DR, Yi JJ, De Koo LO, Ameri H, Puliafito CA, Kashani AH. Optical coherence tomography angiography of diabetic retinopathy in human subjects. Ophthalmic Surg Lasers Imaging Retina. 2015;46(8):796-805.

42. Ishibazawa A, Nagaoka T, Takahashi A, Omae T, Tani T, Sogawa K, et al. Optical coherence tomography angiography in diabetic retinopathy: a prospective pilot study. Am J Ophthalmol. 2015;160(1):35-44.e1. 
43. Thompson IA, Durrani AK, Patel S. Optical coherence tomography angiography characteristics in diabetic patients without clinical diabetic retinopathy. Eye (Lond). 2019;33(4):648-52.

44. Yu S, Lu J, Cao D, Liu R, Liu B, Li T, et al. The role of optical coherence tomography angiography in fundus vascular abnormalities. BMC Ophthalmol. 2016;16:107.

45. Miwa Y, Murakami T, Suzuma K, Uji A, Yoshitake S, Fujimoto M, et al. Relationship between functional and structural changes in diabetic vessels in optical coherence tomography angiography. Sci Rep. 2016;6:29064.

46. Schwartz DM, Fingler J, Kim DY, Zawadzki RJ, Morse LS, Park SS, et al. Phasevariance optical coherence tomography: a technique for noninvasive angiography. Ophthalmology. 2014;121(1):180-7.

47. Hamada M, Ohkoshi K, Inagaki K, Ebihara N, Murakami A. Visualization of microaneurysms using optical coherence tomography angiography: comparison of OCTA en face, OCT B-scan, OCT en face, FA, and IA images. Jpn J Ophthalmol. 2018;62(2):168-75.

48. Couturier A, Mané V, Bonnin S, Erginay A, Massin P, Gaudric A, et al. Capillary plexus anomalies in diabetic retinopathy on optical coherence tomography angiography. Retina. 2015;35(11):2384-91.

49. Hwang TS, Jia Y, Gao SS, Bailey ST, Lauer AK, Flaxel CJ, et al. Optical coherence tomography angiography features of diabetic retinopathy. Retina. 2015;35(11):2371-6.

50. Nakao S, Yoshida S, Kaizu Y, Yamaguchi M, Wada I, Ishibashi T, et al. Microaneurysm detection in diabetic retinopathy using OCT angiography may depend on intramicroaneurysmal turbulence. Ophthalmol Retina. 2018; 2(11):1171-3.

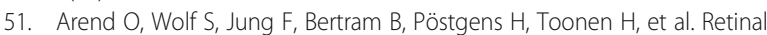
microcirculation in patients with diabetes mellitus: dynamic and morphological analysis of perifoveal capillary network. Br J Ophthalmol. 1991;75(9):514-8.

52. Schreur V, Domanian A, Liefers B, Venhuizen FG, Klevering BJ, Hoyng CB, et al. Morphological and topographical appearance of microaneurysms on optical coherence tomography angiography. Br J Ophthalmol. 2018;103(5): 630-5.

53. Parravano M, De Geronimo D, Scarinci F, Querques L, Virgili G, Simonett JM, et al. Diabetic microaneurysms internal reflectivity on spectral-domain optical coherence tomography and optical coherence tomography angiography detection. Am J Ophthalmol. 2017:179:90-6.

54. Schaal KB, Munk MR, Wyssmueller I, Berger LE, Zinkernagel MS, Wolf S. Vascular abnormalities in diabetic retinopathy assessed with swept-source optical coherence tomography angiography widefield imaging. Retina. 2019;39(1):79-87.

55. de Carlo TE, Bonini Filho MA, Baumal CR, Reichel E, Rogers A, Witkin AJ, et al. Evaluation of preretinal neovascularization in proliferative diabetic retinopathy using optical coherence tomography angiography. Ophthalmic Surg Lasers Imaging Retina. 2016;47(2):115-9.

56. Pan J, Chen D, Yang X, Zou R, Zhao K, Cheng D, et al. Characteristics of neovascularization in early stages of proliferative diabetic retinopathy by optical coherence tomography angiography. Am J Ophthalmol. 2018;192:146-56.

57. Alam M, Zhang Y, Lim Jl, Chan RVP, Yang M, Yao X. Quantitative optical coherence tomography angiography features for objective classification and staging of diabetic retinopathy. Retina. 2018. https://doi.org/10.1097/IAE. 0000000000002373.

58. Lee $H$, Lee $M$, Chung $H$, Kim HC. Quantification of retinal vessel tortuosity in diabetic retinopathy using optical coherence tomography angiography. Retina. 2018:38(5):976-85

59. Uji A, Balasubramanian S, Lei J, Baghdasaryan E, Al-Sheikh M, Sadda SR. Impact of multiple en face image averaging on quantitative assessment from optical coherence tomography angiography images. Ophthalmology. 2017;124(7):944-52.

60. Anegondi N, Kshirsagar A, Mochi TB, Sinha RA. Quantitative comparison of retinal vascular features in optical coherence tomography angiography images from three different devices. Ophthalmic Surg Lasers Imaging Retina. 2018;49(7):488-96.

61. Schottenhamml J, Moult EM, Ploner S, Lee B, Novais EA, Cole E, et al. An automatic, intercapillary area-based algorithm for quantifying diabetesrelated capillary dropout using optical coherence tomography angiography. Retina. 2016;36(Suppl 1):S93-S101.

62. Pedinielli A, Bonnin S, El Sanharawi M, Mané V, Erginay A, Couturier A, et al. Three different optical coherence tomography angiography measurement methods for assessing capillary density changes in diabetic retinopathy. Ophthalmic Surg Lasers Imaging Retina. 2017;48(5):378-84.

63. Lu Y, Simonett JM, Wang J, Zhang M, Hwang T, Hagag AM, et al. Evaluation of automatically quantified foveal avascular zone metrics for diagnosis of diabetic retinopathy using optical coherence tomography angiography. Invest Ophthalmol Vis Sci. 2018:59(6):2212-21.

64. Lee MW, Kim KM, Lim HB, Jo YJ, Kim JY. Repeatability of vessel density measurements using optical coherence tomography angiography in retinal diseases. Br J Ophthalmol. 2018;103(5):704-10.

65. Munk MR, Giannakaki-Zimmermann H, Berger L, Huf W, Ebneter A, Wolf S, et al. OCT-angiography: a qualitative and quantitative comparison of $4 \mathrm{OCT}$ A devices. PLoS One. 2017;12(5):e0177059.

66. Tang FY, Ng DS, Lam A, Luk F, Wong R, Chan C, et al. Determinants of quantitative optical coherence tomography angiography metrics in patients with diabetes. Sci Rep. 2017;7(1):2575

67. You Q, Freeman WR, Weinreb RN, Zangwill L, Manalastas PIC, Saunders LJ, et al. Reproducibility of vessel density measurement with optical coherence tomography angiography in eyes with and without retinopathy. Retina. 2017:37(8):1475-82.

68. Durbin MK, An L, Shemonski ND, Soares M, Santos T, Lopes M, et al. Quantification of retinal microvascular density in optical coherence tomographic angiography images in diabetic retinopathy. JAMA Ophthalmol. 2017;135(4):370-6.

69. Dimitrova G, Chihara E, Takahashi H, Amano H, Okazaki K. Quantitative retinal optical coherence tomography angiography in patients with diabetes without diabetic retinopathy. Invest Ophthalmol Vis Sci. 2017;58(1):190-6.

70. Al-Sheikh M, Akil H, Pfau M, Sadda SR. Swept-source oct angiography imaging of the foveal avascular zone and macular capillary network density in diabetic retinopathyoct-angiography in diabetic retinopathy. Invest Ophthalmol Vis Sci. 2016;57(8):3907-13.

71. Carnevali A, Sacconi R, Corbelli E, Tomasso L, Querques L, Zerbini G, et al. Optical coherence tomography angiography analysis of retinal vascular plexuses and choriocapillaris in patients with type 1 diabetes without diabetic retinopathy. Acta Diabetol. 2017;54(7):695-702.

72. Cicinelli MV, Carnevali A, Rabiolo A, Querques L, Zucchiatti I, Scorcia V, et al. Clinical spectrum of macular-foveal capillaries evaluated with optical coherence tomography angiography. Retina. 2017;37(3):436-43.

73. Ting DSW, Tan GSW, Agrawal R, Yanagi Y, Sie NM, Wong CW, et al. Optical coherence tomographic angiography in type 2 diabetes and diabetic retinopathyoct angiography in type 2 diabetes and diabetic retinopathyoct angiography in type 2 diabetes and diabetic retinopathy. JAMA Ophthalmol. 2017;135(4):306-12.

74. Bhanushali D, Anegondi N, Gadde SG, Srinivasan P, Chidambara L, Yadav $N K$, et al. Linking retinal microvasculature features with severity of diabetic retinopathy using optical coherence tomography angiographyretinal vasculature changes in diabetic retinopathy. Invest Ophthalmol Vis Sci. 2016, 57(9):OCT519-25.

75. Zhang M, Hwang TS, Dongye C, Wilson DJ, Huang D, Jia Y. Automated quantification of nonperfusion in three retinal plexuses using projectionresolved optical coherence tomography angiography in diabetic retinopathy. Invest Ophthalmol Vis Sci. 2016;57(13):5101-6.

76. Alibhai AY, De Pretto LR, Moult EM, Or C, Arya M, McGowan M, et al. Quantification of retinal capillary nonperfusion in diabetics using wide-field optical coherence tomography angiography. Retina. 2018. https://doi.org/ 10.1097/IAE.0000000000002403.

77. Cheung CY, Zheng Y, Hsu W, Lee ML, Lau QP, Mitchell P, et al. Retinal vascular tortuosity, blood pressure, and cardiovascular risk factors. Ophthalmology. 2011;118(5):812-8.

78. Sasongko MB, Wong TY, Nguyen TT, Cheung CY, Shaw JE, Wang JJ. Retinal vascular tortuosity in persons with diabetes and diabetic retinopathy. Diabetologia. 2011;54(9):2409-16.

79. Huang F, Dashtbozorg B, Zhang J, Bekkers E, Abbasi-Sureshjani S, Berendschot TTJM, et al. Reliability of using retinal vascular fractal dimension as a biomarker in the diabetic retinopathy detection. J Ophthalmol. 2016;2016:6259047.

80. Zahid S, Dolz-Marco R, Freund KB, Balaratnasingam C, Dansingani K, Gilani F, et al. Fractal dimensional analysis of optical coherence tomography angiography in eyes with diabetic retinopathy. Invest Ophthalmol Vis Sci. 2016;57(11):4940-7.

81. Chen Q, Ma Q, Wu C, Tan F, Chen F, Wu Q, et al. Macular vascular fractal dimension in the deep capillary layer as an early indicator of 
microvascular loss for retinopathy in type 2 diabetic patientsmicrovascular fractals in type 2 diabetic patients. Invest Ophthalmol Vis Sci. 2017;58(9):3785-94.

82. Michalska-Małecka K, Heinke KA. Optical coherence tomography angiography in patients with diabetic retinopathy treated with anti-VEGF intravitreal injections: case report. Medicine (Baltimore). 2017:96(45):e8379.

83. Bhardwaj S, Tsui E, Zahid S, Young E, Mehta N, Agemy S, et al. Value of fractal analysis of optical coherence tomography angiography in various stages of diabetic retinopathy. Retina. 2018;38(9):1816-23.

84. Johannesen SK, Viken JN, Vergmann AS, Grauslund J. Optical coherence tomography angiography and microvascular changes in diabetic retinopathy: a systematic review. Acta Ophthalmol. 2019:97(1):7-14.

85. Salz DA, de Carlo TE, Adhi M, Moult E, Choi W, Baumal CR, et al. Select features of diabetic retinopathy on swept-source optical coherence tomographic angiography compared with fluorescein angiography and normal eyes. JAMA Ophthalmol. 2016;134(6):644-50.

86. Garcia JM, Lima TT, Louzada RN, Rassi AT, Isaac DL, Avila M. Diabetic macular ischemia diagnosis: comparison between optical coherence tomography angiography and fluorescein angiography. J Ophthalmol. 2016; 2016:3989310

87. Nesper PL, Scarinci F, Fawzi AA. Adaptive optics reveals photoreceptor abnormalities in diabetic macular ischemia. PLoS One. 2017;12(1):e0169926.

88. Dodo Y, Suzuma K, Ishihara K, Yoshitake S, Fujimoto M, Yoshitake T, et al. Clinical relevance of reduced decorrelation signals in the diabetic inner choroid on optical coherence tomography angiography. Sci Rep. 2017;7(1):5227.

89. Yasukura S, Murakami T, Suzuma K, Yoshitake T, Nakanishi H, Fujimoto M, et al. Diabetic nonperfused areas in macular and extramacular regions on wide-field optical coherence tomography angiography. Invest Ophthalmol Vis Sci. 2018;59(15):5893-903.

90. Yasin Alibhai A, Moult EM, Shahzad R, Rebhun CB, Moreira-Neto C, McGowan $M$, et al. Quantifying microvascular changes using OCT angiography in diabetic eyes without clinical evidence of retinopathy. Ophthalmol Retina. 2018;2(5):418-27.

91. Musat O, Cernat C, Labib M, Gheorghe A, Toma O, Zamfir M, et al. Diabetic macular edema. Rom J Ophthalmol. 2015;59(3):133-6.

92. Lee J, Moon BG, Cho AR, Yoon YH. Optical coherence tomography angiography of dme and its association with anti-vegf treatment response. Ophthalmology. 2016;123(11):2368-75.

93. Toto L, D'Aloisio R, Di Nicola M, Di Martino G, Di Staso S, Ciancaglini M, et al. Qualitative and quantitative assessment of vascular changes in diabetic macular edema after dexamethasone implant using optical coherence tomography angiography. Int J Mol Sci. 2017:18(6):1181.

94. de Carlo TE, Chin AT, Joseph T, Baumal CR, Witkin AJ, Duker JS, et al. Distinguishing diabetic macular edema from capillary nonperfusion using optical coherence tomography angiography. Ophthalmic Surg Lasers Imaging Retina. 2016;47(2):108-14.

95. Spaide RF. Volume-rendered optical coherence tomography of diabetic retinopathy pilot study. Am J Ophthalmol. 2015;160(6):1200-10

96. AttaAllah HR, Mohamed AAM, Ali MA. Macular vessels density in diabetic retinopathy: quantitative assessment using optical coherence tomography angiography. Int Ophthalmol. 2018;39(8):1845-59.

97. Gill A, Cole ED, Novais EA, Louzada RN, de Carlo T, Duker JS, et al. Visualization of changes in the foveal avascular zone in both observed and treated diabetic macular edema using optical coherence tomography angiography. Int J Retina Vitreous. 2017;3:19.

98. Mishra A, Luthra S, Baranwal VK, Parihar JKS. An interesting case of rubeosis iridis with neovascular glaucoma in a young patient. Med J Armed Forces India. 2013;69(2):187-9.

99. Lee WD, Devarajan K, Chua J, Schmetterer L, Mehta JS, Ang M. Optical coherence tomography angiography for the anterior segment. Eye Vis (Lond). 2019:6:4

100. Ang M, Baskaran M, Werkmeister RM, Chua J, Schmidl D, Aranha Dos Santos $\mathrm{V}$, et al. Anterior segment optical coherence tomography. Prog Retin Eye Res. 2018;66:132-56

101. Ang M, Sim DA, Keane PA, Sng CC, Egan CA, Tufail A, et al. Optical coherence tomography angiography for anterior segment vasculature imaging. Ophthalmology. 2015;122(9):1740-7.

102. Roberts PK, Goldstein DA, Fawzi AA. Anterior segment optical coherence tomography angiography for identification of iris vasculature and staging of iris neovascularization: a pilot study. Curr Eye Res. 2017;42(8):1136-42.
103. Brunner M, Romano V, Steger B, Vinciguerra R, Lawman S, Williams B, et al. Imaging of corneal neovascularization: optical coherence tomography angiography and fluorescence angiography. Invest Ophthalmol Vis Sci. 2018;59(3):1263-9.

104. Ang M, Cai Y, Shahipasand S, Sim DA, Keane PA, Sng CC, et al. En face optical coherence tomography angiography for corneal neovascularisation. Br J Ophthalmol. 2016;100(5):616-21.

105. Cai Y, Alio del Barrio JL, Wilkins MR, Ang M. Serial optical coherence tomography angiography for corneal vascularization. Graefes Arch Clin Exp Ophthalmol. 2017;255(1):135-9.

106. Ang M, Cai Y, MacPhee B, Sim DA, Keane PA, Sng CCA, et al. Optical coherence tomography angiography and indocyanine green angiography for corneal vascularisation. Br J Ophthalmol. 2016;100(11):1557-63.

\section{Ready to submit your research? Choose BMC and benefit from:}

- fast, convenient online submission

- thorough peer review by experienced researchers in your field

- rapid publication on acceptance

- support for research data, including large and complex data types

- gold Open Access which fosters wider collaboration and increased citations

- maximum visibility for your research: over $100 \mathrm{M}$ website views per year

At BMC, research is always in progress.

Learn more biomedcentral.com/submissions 